\title{
Primary Renal Ewing Sarcoma: A Case Report and Review of the Literature
}

\author{
Bridget L Findlay ${ }^{1 \#}$, Brian M Shinder ${ }^{1 \#}$, Aisha Fatima ${ }^{2}$, Evita Sadimin ${ }^{2}$, Richard Drachtman ${ }^{3}$, Rahul $R$ \\ Parikh $^{4}$ and Eric A Singer ${ }^{1^{*}}$
}

${ }^{1}$ Section of Urologic Oncology, Rutgers Cancer Institute of New Jersey and Rutgers Robert Wood Johnson Medical School, USA

${ }^{2}$ Section of Urologic Pathology, Rutgers Cancer Institute of New Jersey and Rutgers Robert Wood Johnson Medical School, USA

${ }^{3}$ Section of Pediatric Hematology/Oncology, Rutgers Cancer Institute of New Jersey and Rutgers Robert Wood Johnson Medical School, USA

${ }^{4}$ Department of Radiation Oncology, Rutgers Cancer Institute of New Jersey and Rutgers Robert Wood Johnson Medical School, USA

"Equal authorship contribution

\begin{abstract}
Primary Ewing sarcoma of the kidney is an extremely rare and aggressive tumor affecting young adults. We present the case of a 22-year-old male with primary Ewing sarcoma/primitive neuroectodermal tumor (EWS/PNET) of the kidney who underwent right radical nephrectomy and adjuvant chemo-radiation.
\end{abstract}

Keywords

Renal mass, Ewing's sarcoma, PNET

\section{Introduction}

The differential diagnosis of a renal mass in a young adult includes renal cell carcinoma, Wilms tumor, neuroblastoma, lymphoma, metastatic renal involvement from another primary sight, and, rarely, primary renal sarcoma [1,2]. The Ewing sarcoma family tumors (ESFT), which include Ewing sarcoma of bone, extraosseous Ewing sarcoma, and primitive neuroectodermal tumor (PNET), exhibit highly aggressive malignant behavior. Though Ewing sarcoma (ES) is the second most common malignancy of bone and soft tissue in children and young adults, primary extraosseous ES is quite rare [3]. Indeed, just over 100 reported cases of ES of the kidney has been reported worldwide since the first documented case by Seemayer, et al. in 1975 (Table 1) [1,4]. Here, we present a case of extra-osseous Ewing sarcoma/primitive neuroectodermal tumor (EWS/PNET) of the kidney.

\section{Case Presentation}

A 22-year-old male presented to the Emergency Department of a community medical center with abdominal pain for 1-2 weeks. He denied any hematuria, dysuria, flank pain, fevers, bone pain, anorexia, nausea, vomiting, or weight loss. His past medical history was negative for renal calculi or any chronic illnesses. He endorsed occasional smoking, but denied any hazardous or environmental exposures through his work in construction. An initial evaluation with a non-contrast CT of the abdomen and pelvis revealed a $19 \mathrm{~cm}$ diffusely heterogeneous right renal mass. Further evaluation with an abdominal $\mathrm{MRI}$ confirmed the presence of a right renal mass, measuring $20.3 \times 12.6 \times 10.6 \mathrm{~cm}$, with both solid and cystic components (Figure 1). A chest CT identified multiple sub-centimeter lung nodules. Initial laboratory testing was unremarkable with the exception of a mild leukocytosis and $2+$ blood on urine dipstick.

Given this presentation and high suspicion for renal car-

*Corresponding author: Eric A Singer, MD, MA, FACS, Rutgers Cancer Institute of New Jersey, Rutgers Robert Wood Johnson Medical School, 195 Little, Albany Street Room 4563, New Brunswick, NJ 08901, USA, Tel: 732-235-2043, Fax: 732-2356596

Accepted: May 02, 2019

Published online: May 04, 2019

Citation: Findlay BL, Shinder B, Fatima A, et al. (2019) Primary Renal Ewing Sarcoma: A Case Report and Review of the Literature. J Ren Cancer 2(1):15-19 
Citation: Findlay BL, Shinder B, Fatima A, et al. (2019) Primary Renal Ewing Sarcoma: A Case Report and Review of the Literature. J Ren Cancer 2(1):15-19

Table 1: Selected prior reports of primary Ewing sarcoma of the kidney with the site of metastasis and treatment modalities.

\begin{tabular}{|l|l|l|l|}
\hline Study & Patient age, sex & Metastasis & Treatment \\
\hline Moustafellos P, et al. [15] & 32, M & No & Radical nephrectomy, Adjuvant chemotherapy \\
\hline Fergany AF, et al. [16] & 31, M & Yes - lung & Radical nephrectomy, Adjuvant chemotherapy \\
\hline Lazzara B, et al. [17] & 38, M & No & $\begin{array}{l}\text { Radical nephrectomy, Neoadjuvant chemotherapy, } \\
\text { Adjuvant chemotherapy, Radiation }\end{array}$ \\
\hline Vrdoljak DV, et al. [18] & 51, M & Yes - liver, Right iliac wing & Radical nephrectomy, Adjuvant chemotherapy \\
\hline Almeida MFA, et al. [1] & 19, M & Yes - lung & Radical nephrectomy, Adjuvant chemotherapy \\
\hline Liu Z, et al. [19] & $37, \mathrm{M}$ & No & Radical nephrectomy, Adjuvant chemotherapy \\
\hline Chakrabarti N, et al. [20] & 24, F & Yes - lung & Radical nephrectomy, Adjuvant chemotherapy \\
\hline Nam JK [21] & $30, \mathrm{M}$ & No & Radical nephrectomy, Adjuvant chemotherapy \\
\hline Alasmari F, et al. [22] & 15, F & Yes - lung & $\begin{array}{l}\text { Radical nephrectomy, Adjuvant chemotherapy, } \\
\text { Radiation }\end{array}$ \\
\hline
\end{tabular}

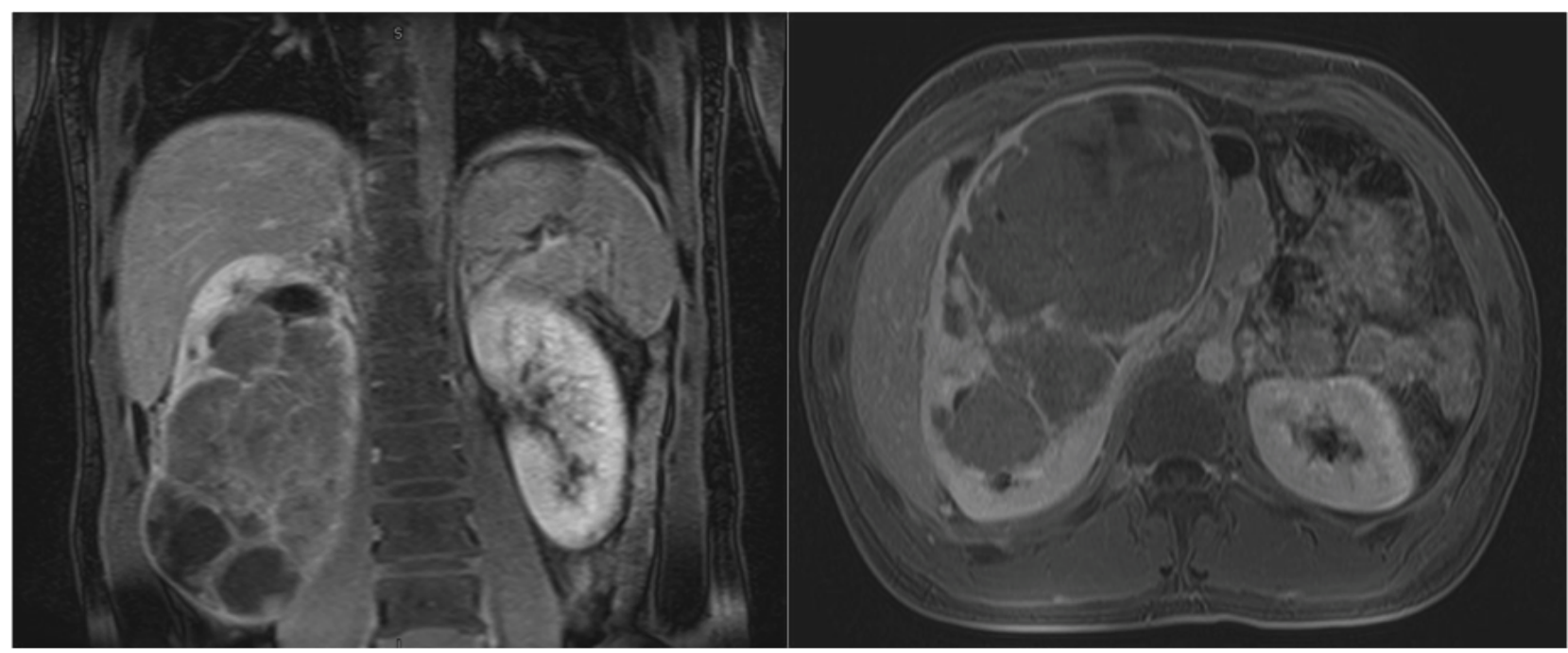

Figure 1: Representative MRI images showing the $20.3 \times 12.6 \times 10.6 \mathrm{~cm}$, with both solid and cystic components.

cinoma, the patient was referred to a $\mathrm{NCl}$-designated comprehensive cancer center for further evaluation and management of his large right renal mass. The patient was reviewed at our multidisciplinary tumor board and Pediatric Hematology/Oncology was asked to participate given the possibility of a Wilms tumor. He subsequently underwent a right open radical nephrectomy and was discharged on post-operative day 2 .

Tumor pathology demonstrated an extraosseous Ewing sarcoma/primitive neuroectodermal tumor with evidence of lymphovascular invasion and microscopic positive tumor at the soft tissue surrounding the vascular margin (R1 resection). Immunohistochemical staining revealed the neoplastic cells positive for CD99 and FLI-1. Cytogenetics showed EWSR1 gene translocation involving the chromosome 22q12. The morphology, immuno-profile and chromosomal aberration support the diagnosis of PNET (Figure 2).

A PET/CT scan performed 2 weeks post-operatively showed residual FDG uptake in the area of the right kidney, but did not demonstrate any other significant hypermetabolic areas. The patient's subcentimeter lung nodules were considered metastatic disease based on clinical staging. A bone marrow biopsy was negative for metastatic disease. The patient enrolled in a clinical trial (NCT02306161) evaluating adjuvant chemotherapy consisting of vincristine/adriamycin/ cyclophosphamide alternated with ifosfamide/etoposide in combination with ganitumab for patients with metastatic Ewing sarcoma. He also received $50.4 \mathrm{~Gy}$ radiation therapy to the right flank in addition to whole lung irradiation (WLI) to 15 $\mathrm{Gy}$ in 10 fractions. In efforts to minimize any overlap between the flank radiation and $\mathrm{WLI}$, as well as minimizing secondary effects of radiotherapy, he was treated with proton beam therapy (PBT) to the flank (Figure 3).

\section{Discussion}

Unlike in renal cell carcinoma, primary renal EWS/PNET is most often a disease of relatively younger patients. The mean age at diagnosis is just 30.4 years, and more than half of newly diagnosed cases are seen in males $[5,6]$. Common presenting symptoms are similar to that of other renal tumors, and no radiographic features have been identified which are indicative of the pathologic diagnosis [1,2]. Given its aggressive nature, patients typically present with advanced 

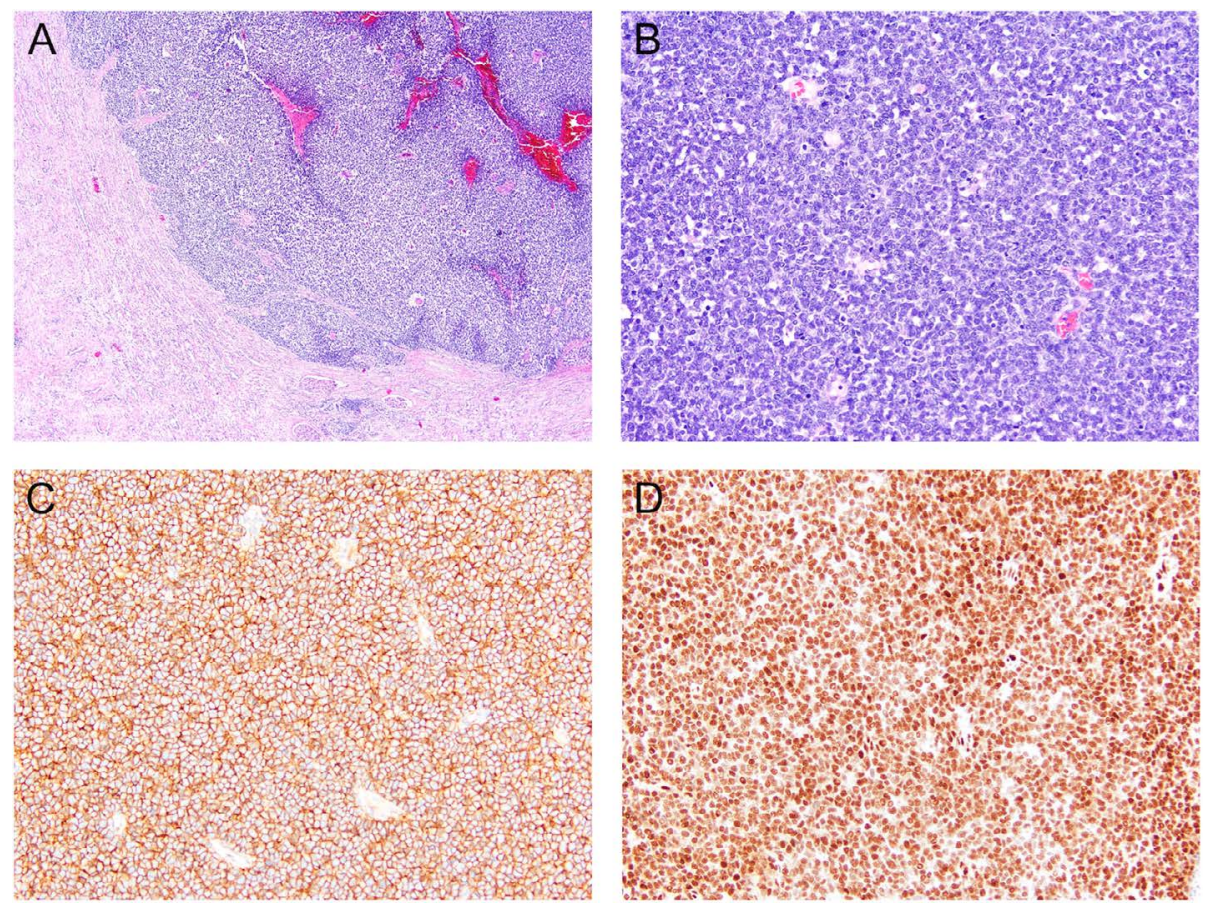

Figure 2: Sheets of monotonous small round blue cells with scant cytoplasm, hyperchromatic nuclei and inconspicuous nucleoli A) Under low magnification (20x) with adjacent renal parenchyma; B) Under higher magnification (200x). The tumor shows strong; C) Membrane staining for CD99 (200x); D) Nuclear staining for FLI-1 (200x).

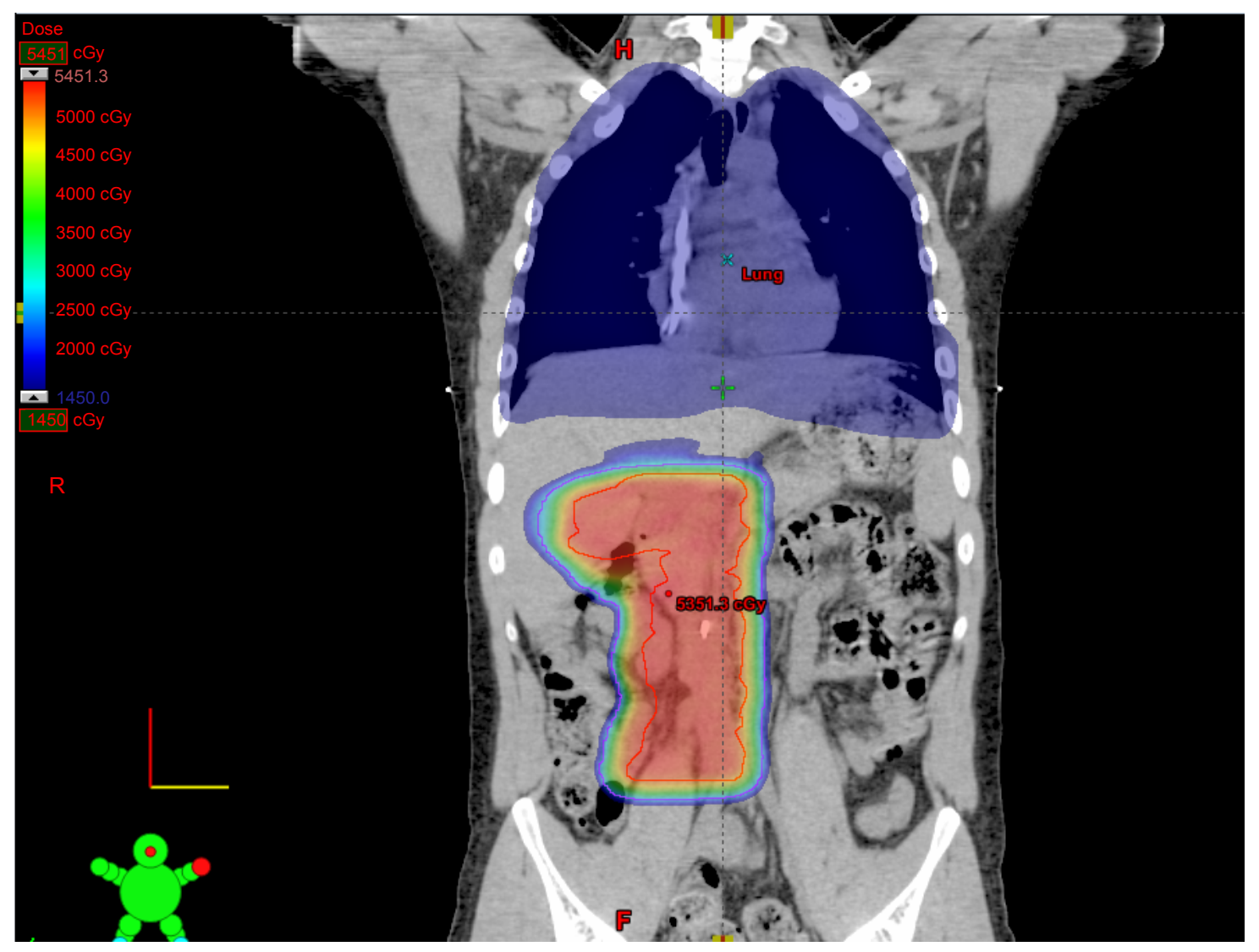

Figure 3: Proton Beam Therapy treatment fields to both the lungs and right flank area, including the resection bed. 


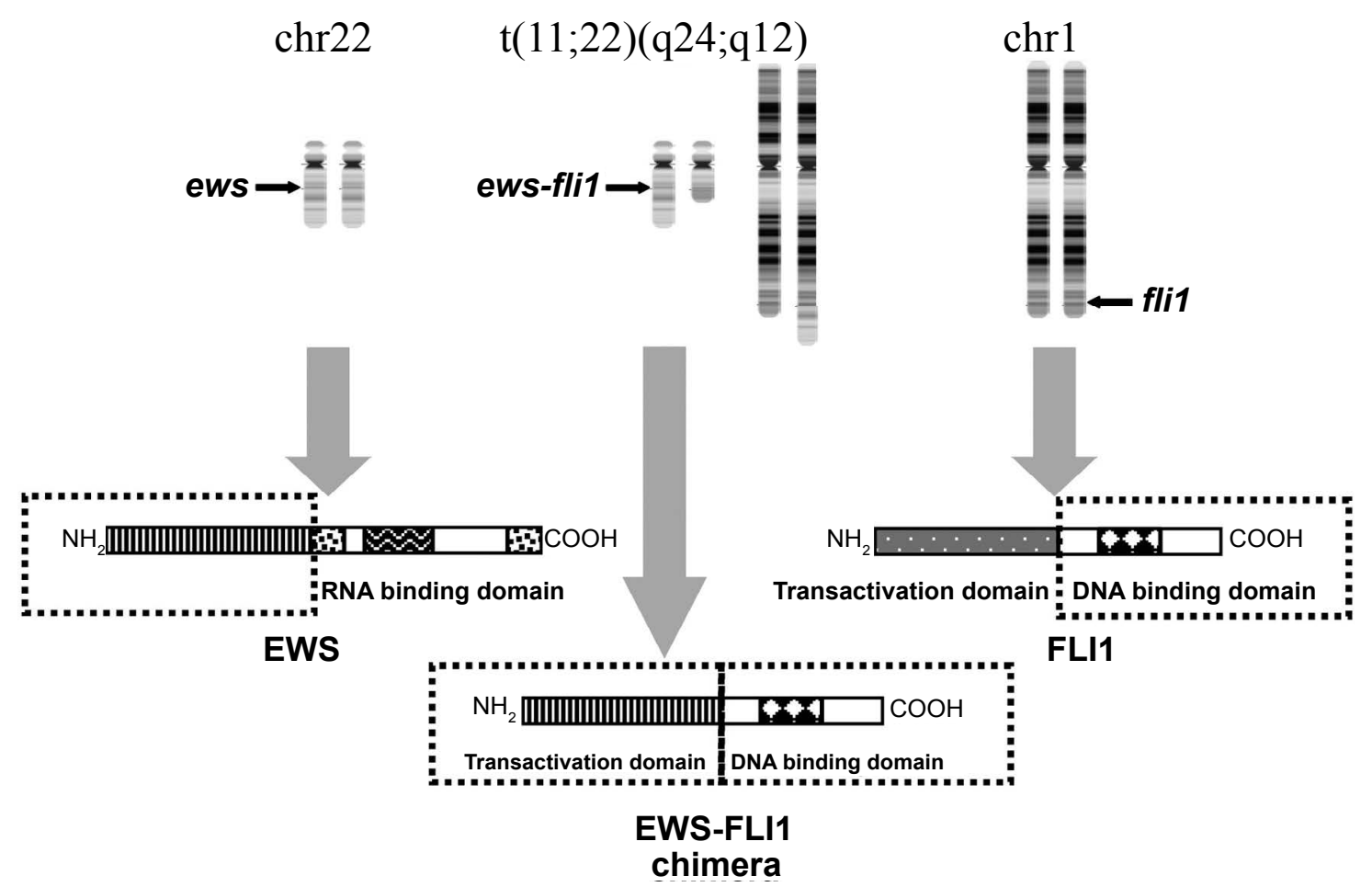

Figure 4: Reciprocal translocation of chromosomes 11 and $22 t(11 ; 22)$ which results in the formation of an EWS-FLI1 fusion gene (Adapted with permission from Bernstein, et al. [10]).

disease and early metastasis [1]. Thus, early and accurate diagnosis of the disease is essential in determining prognosis and establishing a therapeutic strategy.

Immunohistochemistry and molecular studies are necessary in diagnosing Ewing sarcoma family of tumors (ESFT), as they aid in differentiating EWS from other tumors with histologic resemblance, such as Wilms tumor, neuroblastoma, rhabdomyosarcoma, and lymphoma. One typical histological feature of Ewing sarcoma includes vague rosette formations, and CD99 over expression is a non-specific finding present in nearly all ESFTs [2,7-9]. A more unique feature found in 70$90 \%$ of ESFTs is the DNA translocation $\mathrm{t}(11 ; 22)$, which generates the EWS-FL1 fusion oncogene (Figure 4) $[2,5,6,9,10]$. Diagnosis in this patient was made via Fluorescence in situ Hybridization (FISH). At our institution, the break apart FISH is the standard method for diagnostic confirmation of Ewing sarcoma. This method has been shown to be more sensitive and specific compared to RT-PCR, as some cases that are positive by FISH may be negative by RT-PCR [11]. Therefore, we do not perform RT-PCR on cases already confirmed by FISH.

Common sites of metastasis include lung, liver, abdominal lymph nodes, and bone [6]. Prognosis is poor, with a 5-year survival of $60-70 \%$ in non-metastatic disease and $20-30 \%$ in metastatic disease [2,6]. For ESFT, neoadjuvant chemotherapy along with local control and adjuvant chemotherapy are usually performed [10]. Due to the rarity of primary renal EWS/PNET, a standardized treatment protocol has not been established, though these patients are generally treated with regimens for other ESFT. As such, the available literature does suggest a high response rate to chemotherapy, especially in local disease $[5,6,12,13]$.
A study by Zollner, et al. [12] retrospectively analyzed a database of patients enrolled in clinical trials for Ewing's sarcoma over a 30 year period and found 24 patients with primary renal involvement. All patients were treated according to a chemotherapy protocol modeled after established osseous EWS treatments, which consisted of 6 cycles of vincristine, ifosfamide, doxorubicin, and etoposide (VIDE), then risk-adjusted consolidation therapy following local control. The 3 -year overall survival for all those examined was 0.80 , while it was just 0.58 for metastatic disease.

After tumor board review, the patient described in this case was not treated with neoadjuvant therapy due to symptoms from mass effect. However, given the response of ESFT to neoadjuvant treatments, clinicians should have a high index of suspicion for this type of tumor pathology in younger patients with renal masses. While renal biopsies have been somewhat controversial, the benefit of obtaining a tissue diagnosis of renal EWS and starting neoadjuvant treatment may outweigh the risk of biopsy. Rowe, et al. examined an institutional series of 10 patients with renal EWS and showed that 7 of these patients did undergo a biopsy which led to chemotherapy prior to surgical resection [14-22].

\section{Conclusions}

Primary renal EWS/PNET is a rare yet aggressive tumor that affects mostly men in the second to third decade of life, making a high clinical index of suspicion important in this population. Immunohistochemistry is essential to diagnosis given its close histologic resemblance to other renal round cell tumors. Although overall prognosis is poor, early diagnosis and treatment by a multidisciplinary team with multimodal thera- 
py can improve outcomes. Results of ongoing and future clinical trials are needed to optimize therapeutic pathways and therefore all providers are encouraged to discuss clinical trial participation with their patients.

\section{Funding}

This work is supported by a grant from the National Cancer Institute (P30CA072720).

\section{References}

1. Almeida MF, Patnana M, Korivi BR, et al. (2014) Ewing Sarcoma of the kidney: A rare entity. Case Rep Radiol 2014.

2. Ellinger J, Bastian PJ, Hauser S, et al. (2006) Primitive neuroectodermal tumor: Rare, highly aggressive differential diagnosis in urologic malignancies. Urology 68: 257-262.

3. Riggi N, Stamenkovic I (2007) The Biology of Ewing Sarcoma. Cancer Lett 254: 1-10.

4. Seemayer TA, Thelmo WL, Bolande RP, et al. (1975) Peripheral neuroectodermal tumors. Perspect Pediatr Pathol 2: 151-172.

5. Hakky TS, Gonzalvo AA, Lockhart JL, et al. (2013) Primary Ewing Sarcoma of the kidney: A symptomatic presentation and review of the literature. Ther Adv Urol 5: 153-159.

6. Risi E, lacovelli R, Altavilla A, et al. (2013) Clinical and pathological features of primary neuroectodermal tumor/Ewing Sarcoma of the kidney. Urology 82: 382-386.

7. Parham DM, Roloson GJ, Feely M, et al. (2001) Primary malignant neuroepithelial tumors of the kidney: A clinicopathologic analysis of 146 adult and pediatric cases from the National Wilms' Tumor Study Group Pathology Center. Am J Surg Pathol 25: 133-146.

8. Celli R, Cai G (2016) Ewing Sarcoma/Primitive Neuroectodermal Tumor of the Kidney: A Rare and Lethal Entity. Arch Pathol Lab Med 140: 281-285.

9. Murugan P, Rao P, Tamboli P, et al. (2018) Primary Ewing Sarcoma/ Primitive Neuroectodermal Tumor of the Kidney: A Clinicopathologic Study of 23 Cases. Pathol Oncol Res 24: 153-159.

10. Bernstein M, Kovar H, Paulussen M, et al. (2006) Ewing's Sarcoma family of tumors: Current management. Oncologist 11: 503-519.
11. Bridge RS, Rajaram V, Dehner LP, et al. (2006) Molecular diagnosis of Ewing Sarcoma/primitive neuroectodermal tumor in routinely processed tissue: A comparison of two FISH strategies and RTPCR in malignant round cell tumors. Mod Pathol 19: 1-8.

12. Zollner S, Dirksen U, Jurgens H, et al. (2013) Renal Ewing tumors. Ann Oncol 24: 2455-2461.

13. Grier HE, Krailo MD, Tarbell NJ, et al. (2003) Addition of ifosfamide and etoposide to standard chemotherapy for Ewing's Sarcoma and primitive neuroectodermal tumor of bone. N Engl J Med 348: 694-701.

14. Rowe RG, Thomas DG, Schuetze SM, et al. (2013) Ewing Sarcoma of the kidney: Case series and literature review of an often overlooked entity in the diagnosis of primary renal tumors. Urology 81: 347-353.

15. Moustafellos P, Gourgiotis S, Athanasopoulos G, et al. (2007) A spontaneously ruptured primitive neuroectodermal tumor/ extraosseous Ewing's Sarcoma of the kidney with renal vein tumor thrombus. Int Urol Nephrol 39: 393-395.

16. Fergany AF, Dhar N, Budd GT, et al. (2009) Primary extraosseous Ewing Sarcoma of the kidney with level III inferior vena cava thrombus. Clin Genitourin Cancer 7: E95-E97.

17. Lazzara BM, Scalcione LR, Garnet DJ, et al. (2012) Radiologypathology conference: Primary perinephric and renal extraosseous Ewing's Sarcoma. Clin Imaging 36: 77-79.

18. Vrdoljak DV, Sitic S, Stanec M, et al. (2012) Primary Ewing's Sarcoma of the kidney: A case report. Coll Antropol 36: 673-676.

19. Liu Z, Wang X, Lu Y, et al. (2014) Primary Ewing Sarcoma/primitive neuroectodermal tumor of the renal pelvis: A case report. World J Surg Oncol 12: 293.

20. Chakrabarti N, Dewasi N, Das S, et al. (2015) Primary Ewing's Sarcoma/Primitive Neuroectodermal Tumor of of Kidney - A Diagnostic Dilemma. Iran J Cancer Prev 8: 129-133.

21. Nam JK (2015) Primary Ewing Sarcoma of the Kidney with Inferior Vena Cava Invasion. Med Surg Urol 3: 1-3.

22. Alasmari F, Albadawe H, Alkhateeb S, et al. (2017) Primary Ewing's Sarcoma of the kidney: A case report. Int J Surg Case Rep 41: 65-67.

DOI: $10.36959 / 896 / 603$

Copyright: (C) 2019 Findlay BL, et al. This is an open-access article distributed under the terms of the Creative Commons Attribution License, which permits unrestricted use, distribution, and reproduction in any medium, provided the original author and source are credited. 